\title{
ATUALIZAÇÃO DE PROFISSIONAIS PARA A PRÁTICA DE EDUCAÇÃO EM SAÚDE COM GRUPOS DE IDOSOS*
}

\section{PROFESSIONAL UPDATE FOR PRACTICE OF HEALTH EDUCATION WITH GROUPS OF ELDERLY}

\section{ATUALIZACIÓN DE PROFESIONALES PARA LA PRACTICA DE EDUCACIÓN EN SALUD CON GRUPOS DE ANCIANOS}

\author{
Recebido: 05/11/2014 \\ Aprovado: 19/03/2015
}

\author{
Álvaro da Silva Santos ${ }^{1}$ \\ Francielle T. N. F. de Mendonça ${ }^{2}$ \\ Danielle Duarte Silva ${ }^{3}$ \\ Mariana Campos de Sousa ${ }^{4}$ \\ Letícia de Lima Pacífico ${ }^{5}$ \\ Michelle H. P. de Paiva 6
}

O objetivo deste trabalho é descrever a experiência na realização de um curso de atualização para profissionais da atenção primária sobre grupos de educação em saúde com idosos. Trata-se de um estudo do tipo relato de experiência, que descreve a vivência de docentes, mestrandos e acadêmicos na implementação de um curso de capacitação integrante do projeto "Educação em Saúde para Idosos: necessidades de capacitação dos profissionais de saúde e criação de grupos no município de Uberaba”. Participaram 98 profissionais. 0 curso aconteceu no período de maio a agosto de 2014. Os temas abordados incluíram aspectos organizacionais das ações de educação em saúde, técnicas ludopedagógicas, temas de interesse para os idosos e principais agravos de saúde na terceira idade. 0 curso de capacitação proporcionou um espaço de interação dialógica entre os participantes, facilitando as discussões sobre o tema e possibilitando o incremento da forma de atuação nas práticas educativas.

Descritores: Educação em Saúde; Idoso; Capacitação Profissional.

The aim of this paper is to describe the experience in conducting a refresher course for primary care professionals about health education groups with elderly people. It is a study of the type case studies, that describes the experience of faculty, graduate students and scholars in the implementation of a training course of the project "Health education for the elderly: needs for training of health professionals and creating groups in the city of Uberaba, Minas Gerais, Brazil." Participated 98 professionals. The stroke happened in the period from May to August 2014. The topics covered included organizational aspects of health education, ludopedagogic techniques, topics of interest to seniors and major health aggravations in the third age. The training course provided an area of dialogical interaction among participants, facilitating discussions on the subject and enabling the increase the form of expertise in educational practices.

Descriptors: Health education; Aged; Professional training.

El objetivo de este trabajo es describir la experiencia en la realización de un curso de actualización para profesionales de atención primaria acerca de los grupos de Educación de salud con las personas mayores. Es un estudio del tipo relacto de experiencia, que describe la vivencia de profesores, estudiantes de graduación y pós grado en la implementación de un curso de capacitación del proyecto "Educación en salud para las personas mayores: necesidades de capacitación de profesionales de salud y creación de grupos en la ciudad de Uberaba, Minas Gerais, Brazil." Participarón 98 profesionales. El curso ocurrió en el período de mayo a agosto de 2014. Los temas tratados incluyeron aspectos organizativos de la educación en salud, técnicas ludopedagógicas, temas de interés para las personas mayores y principales agravios de la salud en la tercera edad. El curso proporciono una interacción dialógica entre los participantes, facilitando las discusiones sobre el tema, lo que permitio el aumento de conocimientos para las prácticas educativas.

Descriptores: Educación en salud, Anciano; Capacitación profesional.

\footnotetext{
1Enfermeiro. Especialista em Saúde Pública. Mestre em Administração em Serviços de Saúde. Doutor em Ciências Sociais. Pós Doutorado em Serviço Social. Professor Adjunto de Graduação e do Mestrado em Atenção à Saúde da Universidade Federal do Triângulo Mineiro (UFTM). alvaroenf@hotmail.com

${ }^{2}$ Enfermeira. Especialista pela Residência Multiprofissional em Saúde em Saúde do Adulto. Mestranda do Programa de Pós-Graduação em Atenção à Saúde da UFTM.francielletoniolo.enf@gmail.com

${ }^{3}$ Graduanda em Medicina pela Universidade Federal do Triângulo Mineiro (UFTM). Bolsista de Iniciação Científica pelo CNPq. danielleduarte92@yahoo.com.br

${ }^{4}$ Enfermeira. Bolsista de Iniciação Científica pela FAPEMIG. mariana_camposdesousa@hotmail.com

${ }^{5}$ Graduanda em Enfermagem pela Universidade Federal do Triângulo Mineiro (UFTM). Bolsista de Iniciação Científica pela FAPEMIG.

${ }^{6}$ Terapeuta Ocupacional. Especialista pela modalidade Residência Multiprofissional na área de Saúde do Idoso. Mestra em Atenção à Saúde pela Universidade Federal do Triângulo Mineiro (UFTM).

* Este artigo é parte do Projeto intitulado Educação em Saúde para Idosos: necessidade de capacitação dos profissionais de saúde e criação de grupos educativos no município de Uberaba/ Minas Gerais, financiado pela Fundação de Apoio a Pesquisa de Minas Gerais (FAPEMIG) na modalidade Extensão e Pesquisa, sob número APQ: 02554-12.
} 


\section{INTRODUÇÃO}

$\mathrm{s}$ cenários de atuação dos $\mathrm{O}$ profissionais da saúde são os mais diversos, com rápido desenvolvimento de novas tecnologias e mudanças relacionadas às necessidades de saúde das pessoas. Para estarem sempre aptos a atuarem de maneira a garantir a integralidade do cuidado e a resolubilidade do sistema, constantemente são necessários a esses profissionais novos conhecimentos ${ }^{1}$.

Sabe-se que em diversos países a população idosa está aumentando. Segundo estimativas entre os anos de 2000 e 2020 o número de idosos duplicará no Brasil, passando de 14,2 para 29,3 milhões, e em 2060 atingirá 73,5 milhões, representando mais de um terço de toda a população².

Com o aumento desta população, as doenças próprias do envelhecimento passaram a ganhar maior expressão no conjunto da sociedade, tendo como resultado uma maior procura pelos serviços de saúde diante destas situações ${ }^{3}$. Portanto, a transição demográfica observada nas últimas décadas no país vem acompanhada de novas necessidades perante os profissionais de saúde.

Desde 2006 a Política Nacional de Saúde da Pessoa Idosa (PNSPI) ${ }^{4}$ determina que os serviços de saúde devem atender às necessidades desta população promovendo a formação e educação permanente dos profissionais na área de saúde da pessoa idosa, além do envelhecimento ativo e saudável para as pessoas.

Para que se atinja esse envelhecimento ativo e saudável, a política recomenda que os profissionais de saúde devam promover e facilitar a participação dos idosos em grupos de terceira idade, atividades que compreendem ações de integração e fortalecimento de vínculo entre os participantes, bem como oportunidade para realização de ações de educação em saúde.

A educação em saúde deve ser entendida como um processo educativo de construção de conhecimentos em saúde que visa à apropriação da temática pela população, tornando-se um conjunto de práticas que contribui para aumentar a autonomia das pessoas no seu cuidado ${ }^{5}$. Desta forma a educação em saúde promove o empoderamento e a autonomia das pessoas em relação à sua própria vida e saúde.

Diversos estudos ${ }^{6-9}$ evidenciam resultados de experiências com grupos de educação em saúde com idosos, e corroboram as recomendações do $\mathrm{PNSPI}^{4}$, mostrando que as atividades em grupo podem contribuir para a valorização da vida, autocuidado, crescimento pessoal e busca ativa da saúde.

Entretanto, a despeito da importância da educação em saúde realizada com foco na promoção da saúde para os idosos, muitas equipes ainda não estão preparadas para o desenvolvimento dessa atividade e mantêm práticas tradicionais de educação em saúde, utilizando metodologia de transmissão de conhecimentos, traduzindo-se em poucos benefícios para a população ${ }^{10-12}$.

Dessa forma, para promover a educação em saúde para a população idosa também é necessário que ocorra a educação voltada para os profissionais de saúde ${ }^{1}$. Assim, o objetivo deste trabalho é descrever a experiência na realização de um curso de atualização para profissionais da atenção primária, sobre grupos de educação em saúde com idosos.

\section{MÉTODO}

Trata-se de um estudo do tipo relato de experiência, que descreve a vivência de docentes, acadêmicos e mestrandos na implementação de um curso de atualização, integrante do projeto "Educação em Saúde para Idosos: necessidades de capacitação dos profissionais de saúde e criação de grupos no município de Uberaba".

0 referido projeto foi realizado no período de setembro de 2013 a janeiro de 2015, subdividido em três fases: a primeira procurou investigar junto aos profissionais da atenção primária temas de interesse para uma capacitação sobre grupos de educação em saúde com idosos; a segunda fase caracterizou-se pela realização da capacitação; e a terceira pelo apoio aos 
profissionais para criação de grupos pilotos. Este trabalho refere-se à segunda fase do projeto - a capacitação com profissionais da Atenção Primária à Saúde (APS) da cidade de Uberaba, propriamente dito.

O curso de capacitação pautou-se na metodologia participativa e dialógica ${ }^{13}$, permitindo a troca de experiências e discussão das possibilidades de implementação das ações em saúde. Os objetivos do curso foram sensibilizar os profissionais de saúde sobre a prática educativa em saúde com idosos, aprimorar e construir novos conhecimentos, e proporcionar espaço de debates e de promoção da aprendizagem com a participação coletiva e multiprofissional.

Para o alcance dos objetivos foram utilizadas dinâmicas de grupo, problematizações e vivências, baseados nos conhecimentos prévios sobre educação em saúde e na realidade prática dos participantes. Além disso, contou-se com o apoio de profissionais das áreas de nutrição, enfermagem, terapia ocupacional, educação física, fisioterapia, medicina e homeopatia, que forneceram um embasamento técnico e objetivo em relação aos assuntos discutidos.

Os encontros aconteceram em salas de aula na Universidade Federal do Triângulo Mineiro (UFTM), às quintas-feiras, das 13:00 às 17:00 horas, no período de maio a agosto de 2014 .

Durante os encontros, as ações desenvolvidas, o comportamento dos participantes e os assuntos discutidos foram registrados em um diário de campo.

0 projeto foi aprovado pelo Comitê de Ética em Pesquisa da UFTM pelo parecer 1658/2010, com auxílio da Fundação de Apoio a Pesquisa de Minas Gerais (FAPEMIG).

\section{RESULTADOS}

Participaram do curso de capacitação profissionais de saúde da APS dos três distritos sanitários da cidade de UberabaMG, totalizando 98 profissionais, divididos em três turmas.

Os temas abordados foram baseados nos resultados da primeira fase do projeto, e após discussão com representantes da Secretaria Municipal de Saúde (SMS) para concordância com os temas. Assim ficaram definidos os tópicos: aspectos organizacionais das ações de educação em saúde, técnicas ludopedagógicas, temas de interesse para os idosos, e principais agravos de saúde na terceira idade. Cada tema foi abordado em um dia, em quatro encontros de quatro horas, totalizando 16 horas de curso para cada turma. Ao final do último encontro foi aplicado um questionário que avaliou a percepção dos participantes sobre a aplicabilidade dos conhecimentos alcançados.

Em todos os dias de curso os participantes foram colocados em círculo para proporcionar um melhor contato e diálogo entre eles.

\section{Primeiro dia da capacitação}

No primeiro dia foram abordados os aspectos organizacionais de ações de educação em saúde com idosos, sendo discutidos os aspectos que fundamentam a criação de grupos (epidemiologia, utilização de bases de dados - IBGE, SIASUS, DATASUS, observação, cultura local, dentre outros); o planejamento (local do grupo, profissionais envolvidos, dia e horário, recursos, temas e divulgação, dentre outros); a implantação (linguagem, interação, abordagem, técnicas, terapia comunitária, dentre outros); e ainda a importância e formas de avaliação do grupo pelos participantes e pelos profissionais (pesquisa qualitativa, grupo focal, entrevista, questionário).

O grupo e as discussões foram mediadas pelo docente responsável pelo curso. No final da explicação sobre os tópicos, os participantes foram divididos em grupos e cada grupo ficou com uma etapa do planejamento da atividade educativa em saúde, conforme havia sido abordado anteriormente. Em seguida, cada grupo apresentou aos outros exemplos de como seria possível a execução daquela etapa em sua unidade de trabalho. As sugestões e idéias de cada grupo foram avaliadas por todos os participantes considerando a realidade de trabalho dos presentes. 
Os participantes relataram algumas dificuldades em planejar os grupos de educação em saúde, principalmente com relação à abordagem dos temas, divulgação dos encontros e uso de técnicas e recursos didáticos.

\section{Segundo dia da capacitação}

No segundo encontro foi apresentado aos participantes algumas técnicas e dinâmicas possíveis de serem aplicadas nos grupos de educação em saúde. Foram discutidos os objetivos e a aplicabilidade de técnicas ludopedagógicas. Os participantes foram previamente divididos em grupos e convidados a apresentarem algumas técnicas extraídas do segundo de um Manual sobre Educação em Saúde, de acesso livre e amplamente usado.

Em seguida, foram feitas reflexões acerca de cada técnica, ressaltando-se os pontos positivos e negativos, juntamente com as possibilidades de adaptação da técnica ao ambiente ao qual vai ser aplicado.

No decorrer do encontro de capacitação foram apresentadas diversas técnicas que ajudam na apresentação, no entrosamento entre os participantes do grupo, e no aquecimento, para iniciar as atividades ou a reflexão sobre determinado tema.

Algumas técnicas eram mais simples, precisavam de poucos materiais e eram fáceis de serem conduzidas. No entanto algumas técnicas requeriam mais materiais, maior tempo e espaço, além de melhor treinamento por parte do coordenador. Além disso, algumas vezes era necessário que a condução da atividade se desse com maior calma e precisão, possibilitando a compreensão e colaboração de todos para que a dinâmica não perdesse seu foco e o objetivo fosse alcançado.

Para a escolha das técnicas utilizadas no curso foram levadas em consideração a composição e objetivos do grupo, que neste caso eram direcionadas à população idosa, por esse motivo foram selecionados técnicas que se adequavam às habilidades dos idosos, que não ofereciam perigo para quedas e nem que pudesse oferecer constrangimento.

\section{Terceiro dia da capacitação}

No terceiro encontro foram apresentados temas diretamente relacionados à população idosa e para os quais há necessidade de discussão. Os temas abordados foram: estimulação cognitiva, orientação temporoespacial, adesão ao tratamento, atividade física e lazer, alimentação e nutrição, e sexualidade, bem como maneiras de lidar com essas condições para auxiliar e promover a qualidade de vida do idoso. Da mesma maneira que no encontro anterior, houve uma divisão prévia dos participantes em grupos, ficando cada grupo responsável por apresentar um tema para os demais colegas.

Foram convidados profissionais que trabalham na área dos temas abordados para acompanhar o encontro. Assim, cada grupo fazia sua apresentação e, logo após, os profissionais frisavam os pontos mais importantes relacionados, e, se necessário, acrescentavam algumas informações não mencionadas.

Quanto à estimulação cognitiva, os grupos apresentaram formas de manter e estimular a cognição dos idosos por meio de atividades lúdicas, leitura e jogos, a maioria confeccionada pelos próprios participantes.

Em relação à orientação temporoespacial, foram dadas sugestões de modificações no ambiente domiciliar, visando diminuir o risco de acidentes. Dentre as orientações estavam: diminuir o uso de tapetes em pisos escorregadios, tomar banho calçado, disponibilizar interruptor próximo à cama e evitar andar no escuro.

Quanto à adesão ao tratamento os grupos responsáveis deram enfoque em dicas para facilitar a organização dos medicamentos e evitar possíveis confusões na hora da medicação, uma vez que a grande maioria dos idosos faz uso de múltiplos fármacos e possuem dificuldade visual e/ou cognitiva. Para essa organização procuraram utilizar materiais de baixo custo disponíveis da unidade de saúde ou no domicílio do paciente, como caixas de papel ou potes plásticos. 
Ao se tratar de atividade física e lazer, foi bastante discutida a importância da prática de uma atividade física de maneira regular e que se adeque aos limites e capacidades de cada idoso, além de proporcionar momentos de lazer, que promovem bem-estar e qualidade de vida.

Quanto à alimentação e nutrição, foram dadas sugestões de como discutir esse assunto em um grupo de idosos, uma vez que é difícil conseguir uma mudança de hábitos alimentares nessa faixa etária. Dentre as propostas apresentadas, uma delas foi realizar um dia de gastronomia, mostrando na prática como é possível mudar a forma de preparo dos alimentos. Outras sugestões incluíram a criação de hortas comunitárias, cozinha experimental, utilização de temperos, visita acompanhada ao supermercado e refeições em conjunto, em que cada participante leva um item para ser consumido junto ao grupo.

0 último tema abordado foi sobre sexualidade na terceira idade e a dificuldade de abordar o tema. Muitas vezes para esta população a forma de pensar a respeito do assunto é tradicionalista e conservadora. Por isso, os participantes que apresentaram o tema, sugeriram que essa abordagem seja feita por profissionais que já tenham vínculo com os idosos, bem como que o momento seja apropriado para discussão do tema, ou seja, após um tempo do grupo, quando as pessoas já estão mais integradas e dispostas a falar sobre assuntos mais delicados.

Os profissionais que participaram da capacitação mostraram-se interessados e empenhados em por em prática as atividades de promoção da saúde com os idosos.

\section{Quarto dia da capacitação}

No quarto encontro o enfoque se deu nos agravos à saúde que mais acometem os idosos e outros temas: demências, doença de Parkinson, doença de Alzheimer, depressão; osteoartrose; hipertensão arterial, diabetes mellitus, cardiopatias; homeopatia e polifarmácia. Novamente houve uma divisão dos participantes em grupos, em que cada grupo ficou responsável por apresentar um dos assuntos. Foram convidados profissionais ligados aos respectivos temas e que auxiliaram nas discussões, cuja ênfase se deu nos principais dados epidemiológicos, complicações e estratégias de abordagem na atenção primária com os idosos.

Buscou-se com este encontro contribuir para o aprimoramento dos conhecimentos acerca desses agravos e formas de manejo junto à população idosa.

Durante os encontros os participantes tinham a oportunidade de dar sua opinião sobre os temas, tirar dúvidas e discutir sobre a possibilidade de aplicar os novos conhecimentos na realidade a qual estão inseridos.

No último encontro de cada turma havia ainda a escolha de duas Unidades Básicas de Saúde para criação dos grupos pilotos de educação em saúde com idosos, terceira fase desta pesquisa.

\section{DISCUSSÃO}

Os encontros se davam da forma mais interativa possível e buscando quebrar modos tradicionais das práticas educativas. 0 uso de círculo e trabalho com pequenos grupos foi a tônica.

0 posicionamento em círculo e os dispositivos de troca de informações e conhecimento favorecem a aprendizagem significativa, caracterizando mais um momento de contínua busca da melhoria da qualidade da assistência prestada pela sua ampla afinidade ao cotidiano dos trabalhadores e dos usuários ${ }^{14}$.

No que se refere às dificuldades da prática foram encontrados resultados similares aos do estudo de Nunes e colaboradores ${ }^{15}$, que investigou as experiências e opiniões de Agentes Comunitários de Saúde (ACS) acerca das atividades educativas em saúde. Verificam que esses profissionais acreditam ser importante a participação dos usuários no planejamento e desenvolvimento dos encontros, procurando sempre discutir assuntos de interesse do grupo, bem como, que o primeiro encontro deve ser bem atraente para mantê-lo no grupo. As principais dificuldades mencionadas foram o local dos encontros, que muitas vezes é de 
propriedade particular e não da própria comunidade, limitando a participação de algumas pessoas por questões particulares; como fazer a divulgação; e como despertar o interesse dos usuários em participar do grupo.

Durante a atividade proposta foi observado uma maior dificuldade dos participantes em relação à última etapa do planejamento: a avaliação do grupo. Identificou-se que os participantes não conheciam quais instrumentos de avaliação existiam e como ou quando poderiam utilizálos.

Como afirmam Motta, Calda e Assis ${ }^{16}$, a avaliação dos encontros é de suma importância, pois permite um retorno positivo ao grupo, com vistas a ajustes contínuos e tem-se a possibilidade de interpretar resultados e identificar o nível alcançado a partir dos objetivos iniciais.

Em um projeto de promoção da saúde realizado na Universidade Estadual do Rio de Janeiro (UERJ), a avaliação foi realizada de forma contínua e sistemática, sendo uma avaliação ao final de cada encontro e uma a cada semestre. No estudo a avaliação junto aos idosos foi feita por meio de um instrumento aberto (um momento marcante, o que mudou em sua vida, o que você mudaria no grupo). Além do registro das respostas, foi realizado um grupo focal para discussão coletiva e qualificação dessas informações. Alguns dados dessa avaliação reforçam as razões da receptividade positiva dos idosos participantes de grupos de promoção da saúde ${ }^{17}$.

No que se refere as técnicas didático pedagógicas se usou como base um dos volumes da coletânea intitulada: "Manual de técnicas pedagógicas e ludopedagógicas para a operacionalização das ações educativas no SUS-São Paulo18". Tal documento de livre acesso tem uma grande quantidade de técnicas que aponta título, público direcionado, número de participantes, cuidados, entre outros. Tem-se como destaque a ludicidade das técnicas e o estímulo a reflexão.

Em estudo realizado na PUC-RS uma equipe de residentes multiprofissionais acompanhou os encontros de um grupo de convivência de idosos de uma Unidade Básica de Saúde (UBS) por um ano, e no ano seguinte elaboraram um plano de oficinas para a promoção da saúde. No desenvolvimento de cada oficina, os residentes buscaram construir materiais didáticos que tivessem $\mathrm{o}$ propósito de ilustrar os temas abordados de forma lúdica e dinâmica, com vistas à melhor compreensão e maior participação dos idosos nas dinâmicas de grupo, fortalecendo o vínculo e promovendo o aprendizado mútuo 6 .

As atividades lúdicas têm obtido grande importância no âmbito da saúde. São por meio das brincadeiras (dinâmicas de grupo ou individual), jogos, arte (pintura, colagem e teatro de fantoches), expressões corporais (esporte e dança), musicoterapia e ludoterapia que não só se alivia o estresse, como se favorece o desenvolvimento dos vínculos afetivos e sociais, positivas condições para se viver em grupo ${ }^{19}$.

As dinâmicas e as técnicas pedagógicas e ludopedagógicas oferecem oportunidades, não só de reflexão e aprofundamento de diferentes situações do dia-a-dia, mas também de criar uma prontidão para a ação, aprimorar o vínculo entre os profissionais e os usuários, e resgatar o "emocional", tão importante quanto às questões racionais e conceituais ${ }^{18}$.

Um aspecto bem enfatizado nas oficinas foi a adequação das técnicas, bem como, que a técnica sozinha pode pouco, se a participação e envolvimento e o estímulo ao interesse do idoso não for atingido.

Dependendo da metodologia e recursos utilizados, o processo pedagógico será facilitado ou dificultado. Por isso, é importante que se busque sempre criar um ambiente democrático e dialógico, favorecendo uma visão mais ampla e crítica da realidade, dos problemas e situações vividas no cotidiano de trabalho ou familiar dos usuários e profissionais envolvidos ${ }^{18}$.

A utilização de técnicas ludopedagógicas e dinâmicas propiciam momentos de prazer, satisfação e alegria; mas, certamente, são momentos que devem 
ser vivenciados com extrema responsabilidade e conduzidos com segurança e comprometimento. Os objetivos que se pretende atingir devem estar muito bem definidos, a fim de não comprometer o encontro $^{18}$. A exemplo disto, o não uso de materiais que possam causar acidentes.

Vários temas ligados à qualidade de vida e saúde foram discutidos e a atividade física e lazer teve importante destaque.

Em um estudo ${ }^{20}$ realizado no interior do estado de São Paulo, os participantes consideraram importante o estímulo às atividades de lazer, especialmente quando se pensa em aumentar as possibilidades de um envelhecimento físico e cognitivo saudável.

0 trabalho com idoso prescinde de interesse e vínculo do profisional, o que se observou na capacitação, além do que a ampla troca de experiências.

Como relatado em outro estudo ${ }^{14}$, observou-se gradativamente um maior entrosamento do grupo e estabelecimento de uma relação horizontal e dialógica entre participantes, profissionais convidados, docentes e mestrandos, facilitando e contribuindo para a troca de informações.

Outro aspecto de destaque é que por vezes os profissionais de saúde conhecem muito sobre uma patologia, mas pouco sobre o que ela representa no coletivo, além do quanto de custos sociais ela alcança, o que se observou neste trabalho.

A importância de conhecer dados epidemiológicos e se discutir sobre as principais causas de adoecimento entre os idosos se dá, pois, como citado por Veras ${ }^{21}$, os fatores de risco para a população idosa já foram identificados. Entretanto apenas isso não é suficiente, uma vez que é necessário saber utilizar esse conhecimento da forma correta.

Ao se chegar no $4^{0}$ dia se verificou uma ampla satisfação nos 3 diferentes grupos, pois, era nítida a ampliação da criatividade e o interesse despertado em levar a experiência para o seu local de trabalho, bem como, a contribuição da instituição de ensino aos serviços.

Assim tal como referido por Torres e colaboradores $^{22}$, esses cursos mostram-se como uma via de mão dupla, caracterizada pela troca de experiência entre o serviço de saúde e a universidade.

Outrossim, como em outros contextos publicados, as atividades e técnicas realizadas auxiliaram no despertar das habilidades pessoais dos profissionais ${ }^{23}$.

\section{CONCLUSÃO}

É crescente a necessidade de grupos de educação em saúde dirigidos aos idosos, uma vez que a população brasileira passa por um processo de envelhecimento, essa faixa etária é a que mais designa cuidados em saúde. Dessa forma os grupos de educação em saúde atuam no sentido de promoção da sáude e prevenção de agravos para essa população.

Ao analisar as atividades realizadas, notou-se o estabelecimento de uma relação horizontal e dialógica entre participantes e profissionais envolvidos, somando-se a uma sensibilização acerca da necessidade da abordagem multiprofissional aos idosos.

Em relação às discussões suscitadas no ambiente da capacitação, o método de direcionamento utilizado pelos facilitadores contribuiu para o surgimento de diversos enfoques e possibilidades, que dependiam principalmente das opiniões pessoais e experiências relatadas pelos participantes. Além disso, ainda contribuiu para as discussões a possibilidade de, a qualquer momento, as atividades serem interrompidas e o participante emitir sua opinião ou sanar sua dúvida.

Outro aspecto a ser mencionado foi quebra do modelo tradicional de transmissão de informações. A divisão em grupos e a preparação dos temas pelos próprios participantes permitiram uma maior inserção deles no ambiente de aprendizado, por meio das atividades práticas e pesquisas por eles desenvolvidas. $\mathrm{Na}$ maioria dos participantes percebeu-se grande comprometimento e envolvimento, os quais se mostraram ávidos por conhecimento e dispostos a mudanças.

As ações de educação em saúde são de grande importância na área da saúde, sobretudo dirigidas aos idosos. 0 curso de 
capacitação proporcionou um espaço de interação dialógica entre os participantes, facilitando as discussões sobre o tema e possibilitando o incremento da forma de atuação nas práticas educativas.

Espera-se que essa capacitação tenha futuramente um impacto positivo para o atendimento aos idosos e o exercício de um trabalho em equipe de forma complementar.

\section{REFERÊNCIAS}

1. Falkenberg MB, Mendes TPL, Moraes EP, Souza EM. Educação em saúde e educação na saúde: conceitos e implicações para a saúde coletiva. Ciênc saúde coletiva [Internet]. mar 2014 [citado em 18 ago 2014]; 19(3):84752. Disponível

em:

http://www.scielosp.org/scielo.php?script= sci_arttext\&pid=S1413-

81232014000300847\&lng=en.

2. Instituto Brasileiro de Geografia e Estatística. Projeção da população do Brasil e Unidades da Federação por sexo e idade para o período 2000-2030 [Internet]. Rio de Janeiro: IBGE; 2013 [citado em 07 jan 2015]. Disponível

em: http://www.ibge.gov.br/home/estatistica/p opulacao/projecao_da_populacao/2013/def ault.shtm.

3. Veras R. Population aging today: demands, challenges and innovations. Rev Saúde Pública [Internet]. 2009 [citado em 18 out 2014];43(3):548-554. Disponível em: http://www.scielo.br/pdf/rsp/v43n3/en_22 4.pdf.

4. Ministério da Saúde (Br). Portaria no 2.528 de 19 de outubro de 2006. Aprova a Política Nacional de Saúde da Pessoa Idosa [Internet]. D.O.U. 20 out 2006 [citado em 07 jan 2015]. Disponível em: http://portal.saude.gov.br/portal/arquivos/ pdf/2528\%20aprova $\% 20$ a $\% 20$ politica $\% 20$ nacional $\% 20 \mathrm{de} \% 20$ saude $\% 20 \mathrm{da} \% 20$ pessoa \%20idosa.pdf.

5. Pinafo E, Nunes EFPA, González AD, Garanhani. Relações entre concepções e práticas de educação em saúde na visão de uma equipe de saúde da família. Trab Educ Saúde. 2011; 9(2):201-21.

6. Serbim AK, Gerlack LF, Marchi DSM, Gaviolli C, Cecconello M, Moreira LB,
Werlang MC. Oficinas multiprofissionais: educação em saúde para idosos de uma comunidade. Rev Eletrônica Gest Saúde [Internet]. 2013 [citado em 01 out 2014]; 4(1):1780-90 Disponível em: http://gestaoesaude.unb.br/index.php/gest aoesaude/article/view/242.

7. Campos CNA, Santos LC, Aquino JM, Moura MR, Monteiro EMLM. Reinventando práticas de enfermagem na educação em saúde: teatro com idosos. Esc Anna Nery Enferm [Internet]. 2012 [citado em 01 out 2014]; 16(3):588-96. Disponível em: http://www.scielo.br/scielo.php?script=sci_ arttext\&pid=S144-

$81452012000300023 \& \operatorname{lng}=e n \& n r m=$ iso.

8. Combinato DS, Dalla Vecchia M, Lopes EG, Manoel RA, Marino HD, Oliveira ACS et al. "Grupos de Conversa": saúde da pessoa idosa na estratégia saúde da família. Psicol Soc. [Internet]. 2010 [citado em 01 out 2014]; 22(3):558-68. Disponível em: http://www.scielo.br/scielo.php?script=sci_ arttext\&pid=S0102-

$71822010000300016 \& \operatorname{lng}=p t \& n r m=i s o$.

9. Pino M, Ricoy MC, Portela J. Diseño, implementación y evaluación de un programa de educación para la salud con personas mayores. Ciênc Saúde Coletiva [Internet]. 2010 [citado em 01 out 2014]; 15(6). Disponível em: http://www.scielosp.org/scielo.php?script= sci_arttext\&pid=S1413-

81232010000600035\&lng=en\&nrm=iso.

10. Alves GG, Aerts D. As práticas educativas em saúde e a Estratégia Saúde da Família. Ciênc Saúde Colet. [Internet]. 2011 [citado em 13 set 2014]; 16(1):319-25. Disponível em: http://www.scielo.br/scielo.php?script=sci_ arttext\&pid=S1413-

81232011000100034\&lng=en\&nrm=iso.

11. Fernandes MTO, Silva LB, Soares SM. Utilização de tecnologias no trabalho com grupos de diabéticos e hipertensos na Saúde da Família. Ciênc Saúde Colet. [Internet]. 2011 [citado em 01 out 2014]; 16: 1331-40 Disponível em: http://www.scielo.br/scielo.php?script=sci_ arttext\&pid=S1413-

$81232011000700067 \& \operatorname{lng}=e n \& n r m=$ iso. 
12. Oliveira SRG, Wendhausen ALP. (Re)significando a educação em saúde: dificuldades e possibilidades da Estratégia Saúde da Família. Trab Educ Saúde [Internet]. 2014 [citado em 01 out 2014]; 12(1):129-47. Disponível em: http://www.scielo.br/scielo.php?script=sci_ arttext\&pid=S1981-

77462014000100008\&lng=en\&nrm=iso.

13. Freire P. Pedagogia da autonomia: saberes necessários à prática educativa. Rio de Janeiro: Paz e Terra; 2011.

14. Rodrigues ACS, Vieira GLC, Torres HC. A proposta da educação permanente em saúde na atualização da equipe de saúde em diabetes mellitus. Rev Esc Enferm USP. 2010; 44(2):531-7.

15. Nunes JM, Oliveira EN, Machado MFAS, Costa PNP, Vieira NFC. A participação de agentes comunitários de saúde em grupo de educação em saúde. Rev Rene. 2012; 13(5):1084-91.

16. Motta LB, Caldas CP, Assis M. A formação de profissionais para a atenção integral à saúde do idoso: a experiência interdisciplinar do NAI - UNATI/UERJ. Ciên Saúde Coletiva. 2008; 13(4):1143-51.

17. Bernardo MHJ. A saúde no diálogo com a vida cotidiana: a experiência do trabalho educativo com idosos no grupo Roda da Saúde. Rev APS. 2009; 12(4):504-9.

18. Secretaria de Estado da Saúde (São Paulo Estado), Centro de Vigilância Epidemiológica "Prof. Alexandre Vranjac", Núcleo de Educação em Saúde. Educação em saúde: coletânea de técnicas. São Paulo: CVE; 2002. v. 2.
19. Bezerra T, Gomes J. O Lúdico e as atividades de educação em saúde bucal: um estudo de caso na Unidade de Saúde da Família do Km 06-Natal/RN. In: Anais do V Congresso Norte-Nordeste de Pesquisa e Inovação [Internet]; 2010; Maceió. Maceió: IFRN; 2010 [citado em $15 \mathrm{dez}$ 2014]. Disponível em: http://connepi.ifal.edu.br/ocs/index.php/co nnepi/CONNEPI2010/paper/viewFile/309/ 220.

20. Santos PL, Foroni PM, Chaves MCF. Atividades físicas e de lazer e seu impacto sobre a cognição no envelhecimento. Medicina (Ribeirão Preto). 2009; 42(1):54-60.

21. Veras RR. Prevenção de doenças em idosos: os equívocos dos atuais modelos. Cad Saúde Pública. 2012; 28(10):1834-40.

22. Torres HC, Amaral MA, Amorim MM, Cyrino AP, Rodstein R. Capacitação de profissionais da atenção primária à saúde para educação em Diabetes Mellitus. Acta Paul Enferm. 2010; 23(6):751-6.

23. Silveira RE, Reis NA, Santos AS, Borges MR, Fonseca AS. Oficinas com professores: educação em saúde para o manejo com adolescentes. Acta Paul Enferm. 2012; 25(N Esp 2):169-74.

\section{CONTRIBUIÇÕES}

Álvaro da Silva Santos participou da redação e revisão do artigo. Danielle Duarte Silva, Francielle T. N. F. de Mendonça, Mariana Campos de Sousa, Letícia de Lima Pacífico e Michelle H. P. de Paiva participaram da redação do artigo. 